\section{Cellular therapies}

\section{EXPANSION WITH IL-15 INCREASES CYTOTOXICITY OF V $\gamma 9 \mathrm{~V} \delta 2$ T CELLS AND IS ASSOCIATED WITH HIGHER LEVELS OF CYTOTOXIC MOLECULES AND T-BET}

${ }^{1}$ Pia Aehnlich*, ${ }^{2}$ Per Thor Straten, ${ }^{1}$ Ana Micaela Carnaz Simoes, ${ }^{1}$ Signe Skadborg ${ }^{1}$ Gitte Olofsson. ${ }^{1}$ Copenhagen University Hospital, Herlev, Denmark; ${ }^{2}$ University of Copenhagen, Herlev, Denmark

Background Adoptive cell therapy (ACT) is an approved treatment option for certain hematological cancers and has also shown success for some solid cancers. Still, benefit and eligibility do not extend to all patients. ACT with $\mathrm{V} \gamma 9 \mathrm{~V} \delta 2 \mathrm{~T}$ cells is a promising approach to overcome this hurdle.

Methods In this study, we explored the effect of different cytokine conditions on the expansion of $\mathrm{V} \gamma 9 \mathrm{~V} \delta 2 \mathrm{~T}$ cells in vitro.

Results We could show that $\mathrm{V} \gamma 9 \mathrm{~V} \delta 2 \mathrm{~T}$ cell expansion is feasible with two different cytokine conditions: (a) 1000U/ml interleukin (IL)-2 and (b) $100 \mathrm{U} / \mathrm{ml}$ IL-2+100U/ml IL-15. We did not observe differences in expansion rate or $\mathrm{V} \gamma 9 \mathrm{~V} \delta 2 \mathrm{~T}$ cell purity between the conditions; however, IL-2/IL-15-expanded V $\gamma 9 \mathrm{~V} \delta 2 \mathrm{~T}$ cells displayed enhanced cytotoxicity against tumor cells, also in hypoxia. While this increase in killing capacity was not reflected in phenotype, we demonstrated that IL-2/IL15 -expanded V $\gamma 9 \mathrm{~V} \delta 2 \mathrm{~T}$ cells harbor increased amounts of perforin, granzyme B and granulysin in a resting state and release more upon activation. IL-2/IL-15-expanded V $\gamma 9 \mathrm{~V} \delta 2 \mathrm{~T}$ cells also showed higher levels of transcription factor T-bet, which could indicate that T-bet and cytotoxic molecule levels confer the increased cytotoxicity.

Conclusions These results advocate the inclusion of IL-15 into ex vivo $\mathrm{V} \gamma 9 \mathrm{~V} \delta 2 \mathrm{~T}$ cell expansion protocols in future clinical studies.

http://dx.doi.org/10.1136/jitc-2020-SITC2020.0764

\section{CONTEXTUAL SECRETION OF NANOSCALE INTERLEUKIN (IL)-12 BY CAR T CELLS FOR THE TREATMENT OF CANCER}

'Zhifen Yang*, ${ }^{1}$ Maggie Bobbins, ${ }^{2}$ Hana Choi, ${ }^{1}$ Ofir Stefanson, ${ }^{1}$ Jin Yang, ${ }^{1}$ Khristina Magallanes, ${ }^{1}$ Bing Wang, ${ }^{3}$ Lei Stanley Qi, ${ }^{1}$ Francesco Marincola. ${ }^{1}$ Refuge Biotechnologies, MENLO PARK, CA, USA; ${ }^{2}$ Refuge, MENLO PARK, CA, USA; ${ }^{3}$ Stanford University, Palo Alto, CA, USA

Background Interleukin(IL)-12 activates $\mathrm{T}$ cells and macrophages pivoting the switch that turns chronic into acute inflammation and results in cancer rejection. However, despite formidable antitumor effects in preclinical models, its clinical utilization is limited by severe systemic toxicity. Here, we present a conditional, antigen-dependent, non-editing CRISPRactivation (CRISPRa) circuit (RB-2-12) that purposefully induces minimally effective doses of IL-12 for autocrine activation of CAR-T.

Methods RB-2-12 is a CAR T cell engineered to express the IL-12 heterodimer via conditional transcription of its two endogenous subunits p35 and p40. The circuit includes a lentiviral constructs encoding an anti-HER2 (4D5) single chain variable fragment, with $\mathrm{CD} 28$ and $\mathrm{CD} 3 \zeta$ co-stimulatory domains linked to a tobacco etch virus (TEV) protease and two single guide RNAs (sgRNA) targeting the promoter region for IL-12A orL-12B. A second constructs encodes linker for activation of $\mathrm{T}$ cells, complexed to nuclease-deactivated/dead Cas9 (dCas9)-VP64-p65-Rta transcriptional activator (VPR) via a TEV-cleavable linker (LdCV). Activation of CAR brings CAR-TEV in proximity to LdCV releasing dCas9 for nuclear localization to the regulatory regions and conditionally and reversibly induce nanoscale expression of the p70 heterodimer. RB-2-12 was compared in vitro to control (cRB-2-12, lacking the IL-12 sgRNAs).

Results RB-2-12 induced autocrine production of low concentrations of IL-12 upon exposure to HER2 + FaDu cancer cells resulting in significantly enhanced production of interferon (IFN)- $\gamma$, cytotoxic activity and proliferation (figure 1a). These effects were comparable to co-culturing conventional HER2specific CAR-T cells with a modified $\mathrm{FaDu}$ cell line expressing high doses of IL-12 (figure 1b).

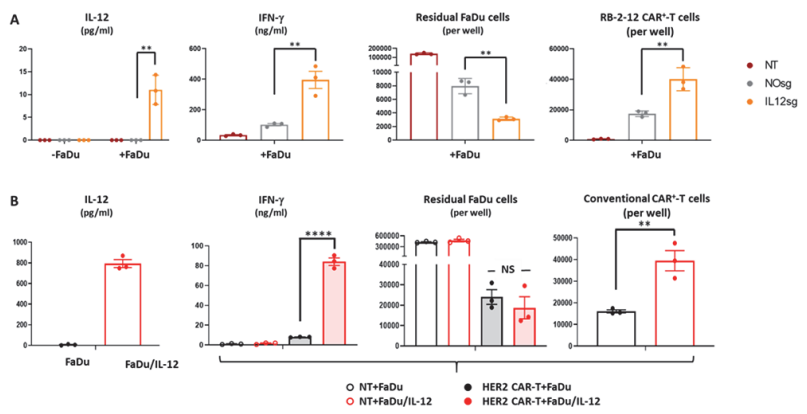

Abstract 765 Figure 1 Conditional autocrine release of nanoscaledose p70/LL-12 by

Conditional autocrine release of nanoscale-dose p70/LL-12 by RB-2-12 resulting in enhanced IFN- $\gamma$ production, cytotoxicity and proliferation in vitro after three days of exposure to FaDu cells (figure 1a). Constitutive high dose release of p70-IL-12 by HER2+ Fadu cells engineered to constitutively express IL-12 (FaDu/IL-12) and its effect on IFN- $\gamma$ secretion, cytotoxicity and proliferation of conventional HER-2-specific CAR T-cells three days after exposure to FaDu or FaDu/lL-12 cancer cells (figure 1b). N.T. = non-transduced T cells; NOsg = cRB-2-12 CAR-T cells missing the sgRNAs for the two IL-12 subunits; IL12sg = complete product incorporating the full CRISPRa functions.

Conclusions We have previously shown that tandem suppression of PD-1 expression upon HER-2 CAR activation using CRISPR interference enhances anti-cancer properties of CAR-T cells in vivo against HER2-FaDu xenografts by promoting their persistence and long-term tumor colonization (companion abstract submitted to SITC annual meeting). We hypothesize that addition of a Th1 polarizing component such as IL-12 will exponentially increase the efficacy of reprogrammed CAR$T$ cells by combining enhancement of effector functions to cellular fitness. At the same time, the autocrine effects of nanoscale IL-12 production limit the risk of off-tumor leakage and systemic toxicity. Such cumulative synthetic biology approaches are currently investigated in vitro and in vivo model systems. Current work is testing the effectiveness of RB-2-12 in vivo against $\mathrm{FaDu}$ xenografts.

Acknowledgements None

Trial Registration N.A.

Ethics Approval Not Applicable

Consent Not Applicable

\section{REFERENCES}

1. None

http://dx.doi.org/10.1136/jitc-2020-SITC2020.0765 